\title{
Supporting Personal Collections across Digital Libraries in Spatial Hypertext
}

\author{
Frank M. Shipman III, Haowei Hsieh, J. Michael Moore, Anna Zacchi \\ Department of Computer Science \\ Texas A\&M University \\ College Station, TX, 77843-3112, USA \\ 1-979-862-3216
}

\{shipman, haowei, jmichael, zacchia\}@cs.tamu.edu

\begin{abstract}
Creating, maintaining, or using a digital library requires the manipulation of digital documents. Information workspaces provide a visual representation allowing users to collect, organize, annotate, and author information. The Visual Knowledge Builder (VKB) helps users access, collect, annotate, and combine materials from digital libraries and other sources into a personal information workspace. VKB has been enhanced to include direct search interfaces for NSDL and Google. Users create a visualization of search results while selecting and organizing materials for their current activity. Additionally, metadata applicators have been added to VKB. This interface allows the rapid addition of metadata to documents and aids the user in the extraction of existing metadata for application to other documents. A study was performed to compare the selection and organization of documents in VKB to the commonly used tools of a Web browser and a word processor. This study shows the value of visual workspaces for such effort but points to the need for subdocument level objects, ephemeral visualizations, and support for moving from visual representations to metadata.
\end{abstract}

\section{Categories and Subject Descriptors}

H.3.7 [Information Storage and Retrieval]: Digital Libraries collection, systems issues, user issues.

\section{General Terms}

Design, Experimentation, Human Factors.

\section{Keywords}

spatial hypertext, incremental formalization, metadata, collection organization, information triage, information visualization

\section{INTRODUCTION}

Libraries are sources of information for many types of activity. They provide a place to locate facts - such as the atomic weight of Cesium, the diameter of the Earth, or a function to compute the

Permission to make digital or hard copies of all or part of this work for personal or classroom use is granted without fee provided that copies are not made or distributed for profit or commercial advantage and that copies bear this notice and the full citation on the first page. To copy otherwise, or republish, to post on servers or to redistribute to lists, requires prior specific permission and/or a fee.

JCDL'04, June 7-11, 2004, Tucson, Arizona, USA.

Copyright 2004 ACM 1-58113-832-6/04/0006...\$5.00. escape velocity from different latitudes. They are also part of longer-term information tasks, such as performing the literature review for a dissertation or designing a new spacecraft. These longer-term information tasks are the focus of our work.

Fischer, Henninger, and Redmiles [4] describe an information life-cycle which includes three phases of activity with a software library: location, comprehension, and modification (or authoring.) Much effort has gone towards addressing the information location problem in digital libraries. The large amounts of information available digitally have driven the need for new techniques for indexing, querying, and visualizing digital resources. For example Rao et al. [12] developed several visualization techniques for browsing retrieved information.

For long-term information tasks, the effort really begins once the source information is located. For these tasks, the comprehension and authoring phases dominate the task. In paper-based libraries, once located, books or articles are checked out or copied to be read somewhere else or read at a desk or table in the library. An advantage for reading in the library is that as new information needs surface, resources are close at hand. Similarly, digital libraries frequently include hypertext links for intertextual or interdocument references. Access to related work is just one way that a library aids the comprehension of its contents - it also provides a physical and social setting in which librarians and other patrons are potential resources.

But this vision is still one of the existing library. The digital library needs to respect the tradition of paper-based libraries while taking advantage of the possibilities available due to new technology. Just as full-text search and metadata visualization extends the tools available to patrons accessing a digital library, users also need interfaces and tools for working with information after it is initially located.

But before going forward, let us return to the traditional library and the workspace it provides. The library reading table or desk is a place where a patron can bring together materials from different parts of the library for personal and task-based reading and interpretation. As one walks through a university library, you see these surfaces combining original source materials (e.g. books and journals), copied and annotated versions of similar materials, and loose paper and notebooks containing patrons' expressions.

Desks and tables include a variety of types of workspaces provided by many libraries. There is the table or desk in the stacks, where one or two people may quietly go about their work. Frequently, small rooms are available for groups to perform tasks requiring the library's resources. Finally, there are tables, desks, 


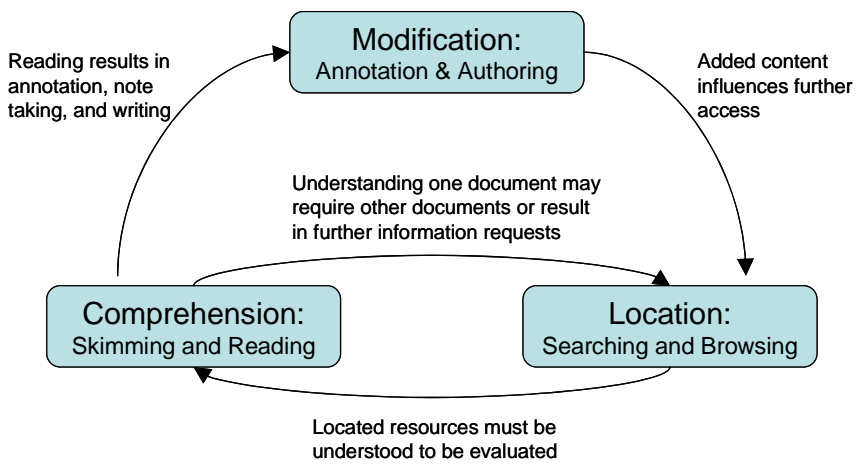

Figure 1: Information Life Cycle

and small and large rooms for the librarians to go about the task of maintaining their collections.

Our work is on the digital version of such workspaces. The next two sections refine Fischer's information life-cycle within the context of digital libraries and present a computational approach for supporting the comprehension and authoring phases of information work. We then describe the Visual Knowledge Builder (VKB), an information workspace that provides such support. This is followed by scenarios of VKB use by library patrons and librarians and some conclusions about digital library workspaces based on our experiences with VKB.

\section{INFORMATION LIFE-CYCLE}

The information life-cycle described by Fischer is in the context of a library of reusable software components. In this context, software developers query the library for software components that may be of use to their current development task. The model expects that patrons of the library, once they understand the located software components, will likely need to modify the components in the library for their particular application. For more general libraries, this notion of modification can be categorized as annotation and authoring. Figure 1 provides an overview of the information life-cycle in a general digital library. An alternative version of this life-cycle for digital libraries with an emphasis on reuse is presented by Sumner and Dawe [19].

Different information tasks begin in different phases of the model. Writing a historical novel may start out as authoring the basic story which later requires the author to locate and comprehend information to create the historically accurate backdrop for the narrative. For the reviewer of papers or proposals, the starting point is comprehension but while reading the materials the reviewer may determine a need to locate related work for comparison. For the student writing a literature review or a term paper or the reference librarian collecting information on a topic, the first step is likely to be locating related work which will then be comprehended and lead to authoring. For the cataloger, the task begins with a set of materials that must be understood to be integrated in an existing collection, and then is followed by modification through the addition of metadata.

An effective digital library must include support for all three phases of the information life-cycle. This does not mean that one interface has to be used for all three tasks, but that applications which support particular phases need to be built with the expectation of users moving back and forth among applications supporting different phases of information work.

Collection management is hidden in this model. Deciding what to include in the library, attaching metadata to these entities, and updating and pruning the materials as time goes by is assumed to be part of modification and authoring. Digital libraries will need annotation, authoring, and publishing practices that match their particular goals and situation. For example, an approach to software collection management promoted by Fischer et al. [5] was seeding, evolutionary growth, and reseeding. This involved an information space that was seeded by experts, then grew through use as people performing real tasks added and modified the materials in the collection, with periods of reseeding when the experts reorganized and checked the consistency of the collection. Such a process is appropriate for a software library when used by trusted software engineers, but differs greatly from the model of a typical public library. However, users could add information and metadata over time and librarians could review the material periodically to reorganize and check for consistency. This is consistent with current trends where users provide feedback that benefits other users such as NSDL.org, DLESE.org, Amazon.com and imdb.com.

\section{APPROACH}

Our emphasis is on tools that support patrons and providers with their comprehension, annotation, and authoring of digital library materials. Our approach provides users with visual information workspaces for collecting, organizing, and authoring information.

The computer desktop is the most common information workspace for most users. The desktop allows users to organize documents into a hierarchy of folders. The documents are represented in the workspace by icons indicating their data storage format or the appropriate application for that format and a document name. Folders within the desktop metaphor are opaque - they are visually represented by a folder icon and most lack any indication of their contents other than their name. Those that do have visual indicators are limited to a few predefined types. Users must open a folder to see and manipulate its contents. Manipulating documents is limited to moving the documents around on the desktop or into a folder, changing the name of a document, and selecting a document type or default application.

The model for our workspace is the physical library table described above. Characteristics of the library table important to the approach include: (1) the integration of original source materials and the interpretation of those materials; (2) the expression of interpretation through implicit and explicit techniques; and (3) the ability for the space to be shared by multiple library patrons.

To provide simultaneous access to library resources and the ability to express interpretations of those resources, a workspace must integrate original library materials with annotated copies of those materials and information authored by the user. Most Webbased libraries expect users to search and view their contents in a Web browser or other document viewer. Users' categorizations and writings are then expressed in a file system structure, a word processor, or other application. While it is simple to move text from on-line sources into a document, other media can pose difficulties. Also, unless an entire document is included, the 
material loses its context and can be difficult to differentiate from other source materials or new writings. This is in contrast to the library table, where published matter is easily differentiated from the patron's notes.

Books, photocopies, and notes are the materials found on the library table but the task of the library patron frequently requires a determination of the interrelationship of these entities. A student writing a literature review in a technical area will collect materials on the topic and then classify them for an orderly exposition. This categorization is done both explicitly, in the form of written notes, and implicitly, in the arrangement of materials on the table. Piles at the university library may be task dependent - those materials needed for a particular assignment or the result of the patron's interpretation, e.g. piles for, against, or ambivalent about a particular concept. In any case, highlighted and annotated copies of text are piled with notebooks and books, all with Post-Its and impromptu placeholders attached.

Library patrons may share a library table, especially if they are working collaboratively on an assignment. The space itself becomes a prop for their conversation. They move and point to objects as they communicate their understanding of their task and the materials. They also use the space to split up work and come to agreement on a strategy for collaboration.

The metaphor of the library table can point towards characteristics of space we want to include in an interface for digital libraries, but we must also determine what opportunities there are for going beyond the physical table. One problem with the library table is that it is a limited resource and so, when the patron leaves, they must pick up and take their materials with them. In this process they lose parts of their interpretive work that are represented by the layout of the items on the tabletop. Additionally, there is often only one instance of each document, a very limited amount of space, and the tabletop can only be in one state at a time.

Finally, the traditional library includes several methods to express relations between and characteristics of materials. There are explicit references between documents as well as connections between documents based on metadata (e.g. documents by the same author or publisher or on the same topic.) Additionally, there is the physical layout of the library, so materials on the same shelf, in the same row, or on the same floor of the library may have some implicit relationship (e.g. the new books section.) Library materials also have size, color, and the effects of age and use. These document characteristics may be of use to patrons and analogues should be included in their digital representations.

\section{THE VISUAL KNOWLEDGE BUILDER}

The Visual Knowledge Builder (VKB) is a spatial hypertext designed to include many of the properties mentioned above. VKB combines the flexible visual workspace found effective for expression in VIKI [10] with the representation and active support for incremental formalization of the Hyper-Object Substrate [17]. The next sections discuss characteristics of the workspace, integrated search, navigable history, and metadata authoring.

\subsection{The Workspace}

The interface to VKB, similar to VIKI, is a two dimensional workspace that contains information objects and other twodimensional spaces, called collections. The goal of VKB's workspace differs from DLITE [2] and other workspaces that visually represent library features to improve usability. Information objects and collections can be resized, positioned, and moved between collections through direct manipulation. Figure 2 shows a workspace created by one of the study subjects.

The user can attach a variety of visual attributes to the information objects and collections to express their interpretation of the materials. The controls for changing the border width, background color, border color, and transparency are on a toolbar that is always available. Additionally, the user may select the font type, size, and color for each object and collection. Similar to the library table, expression of relationships and categories occurs by placing objects near one another or placing objects in a collection.

Navigating into a collection causes the collection to fill the workspace window, similar to Boxer [3] and VIKI. This exposes more of the lower-level workspace, as shown in Figure 2. As with the library table, original source material is interleaved with the student's own notes and interpretation.

Information objects can be augmented with formalized metadata in the form of attribute/value pairs. Attributes can be assigned any name and can be associated with multiple values. Consequently, these attributes and values can be used to create and modify metadata based on the current activity and user desires. Users can add attribute/value pairs by adding text directly to a symbol and following a simple syntax (note the Title and URL attributes in Figure 2) or by using a dialog window. Moreover, types can be created that represent a set of attributes and visual characteristics. Type attributes can be assigned default values. Types can be used to represent commonly co-occurring sets of metadata such as Dublin Core and be applied to information objects.

VKB allows information objects to be associated with external content such as files and URLs, through File and URL attributes respectively. Beyond the methods described above for adding attributes, a drag and drop method can be used for files and URLs. When a URL from a web-browser or a filename from the operating system are dragged into a collection, an information object is created that contains a URL or File attribute for that URL or File. Double clicking on the object will open the URL or File in the appropriate application. If the URL or filename is dragged into an existing object, then the appropriate URL or File attribute is added to that object. This allows an information object to be linked with its source content and viewed or edited in the appropriate interface. More information on basic VKB functionality and its use can be found in [18].

\subsection{Integrated Search}

Observation of VKB use shows many people do searches and then move the URLs into VKB as part of information triage [10] and comprehension. Bypassing this effort would enable users to move more easily from location into comprehension and modification. For this reason, National Science Digital Library (NSDL) [8] and Google searches are integrated into VKB. So users can do their searches and immediately begin processing the results without having to transfer the links as described above.

Search results are presented in a new collection as is also true with the integrated search in VIKI [15] and Garnet [1]. Each result is represented by an information object along with any metadata that can be extracted from the search results. These 


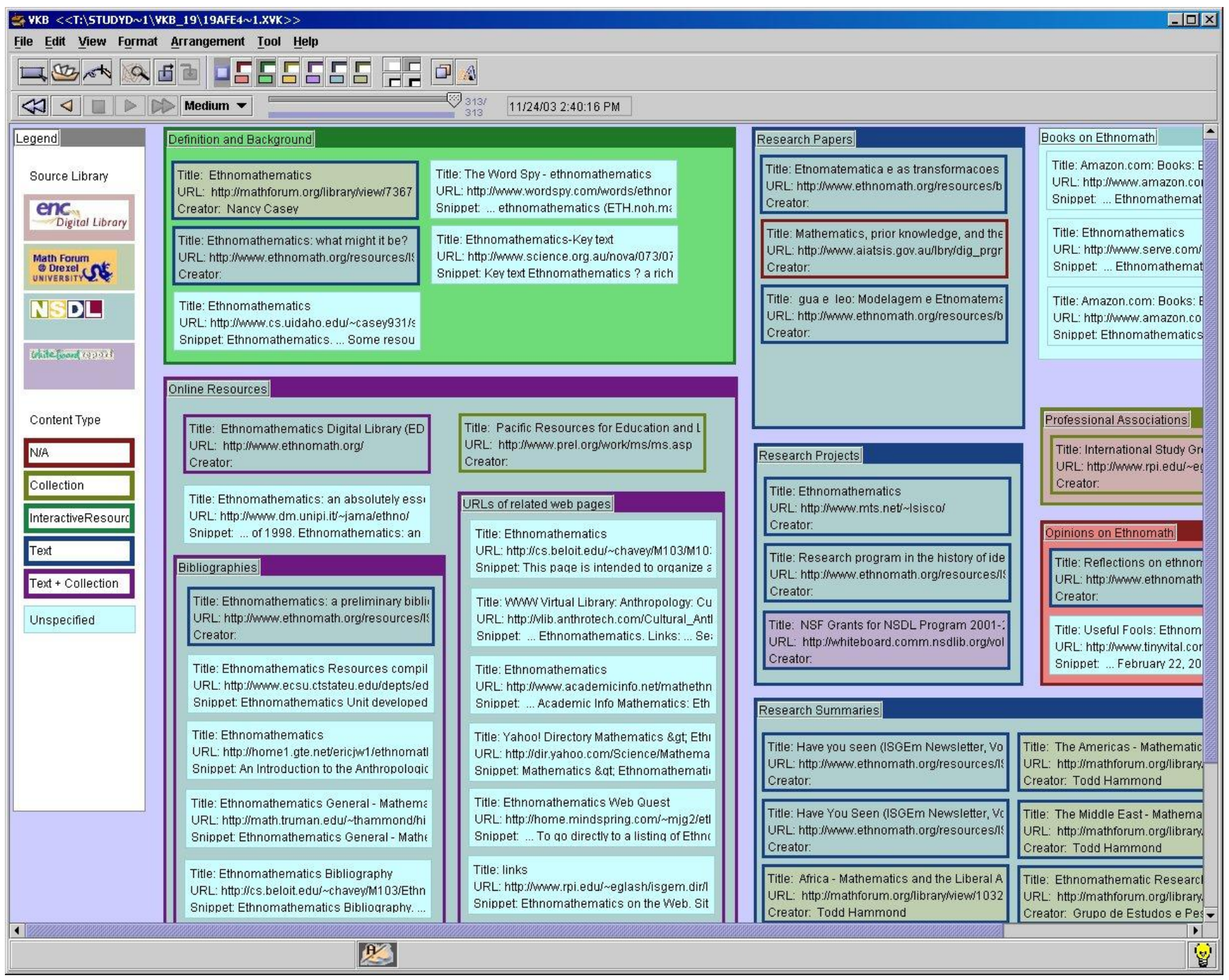

Figure 2: VKB workspace with user interpretation

information objects contain the URLs and metadata of the source documents and are ordered by their search ranking. With NSDL's more comprehensive set of metadata [8], NSDL search results are visually enhanced to convey particular aspects of the metadata. The current visual encoding uses symbol color, border color, and border width to indicate source library, material type (text, image, video, etc), and a number of material types respectively. A legend is generated along with the search results to explain the visual encodings (see left side of Figure 2.)

\subsection{Incremental Metadata}

As workspaces develop over time, the emergent structure and its visual attributes may represent metadata. Users may formalize the interpretations that evolved in the workspace by assigning attributes and values to them. The number of information objects in a space may be large and users may not want to add each attribute/value pair individually to each object. VKB alleviates this in two ways. The first is through a metadata applicator and the other is through the Suggestion Manager.
When users are working, they may find that they are applying the same set of attributes repeatedly. As users identify these sets, they can create a template for applying multiple attributes to an information object or a set of information objects en masse. The Metadata Applicator provides this functionality. An attribute extractor assists users in creating sets of attributes. An information object or set of information objects that already have a desired set of attributes act as a template for creating a set of attributes for the attribute applicator. After some editing, the attribute set is ready to use.

To add attributes to objects with the Metadata Applicator, a symbol or group of objects is selected and pressing the desired application button implements the change (Figure 4). The Metadata Applicator allows different application methods. Users can apply a whole set of attributes at once, or select a particular value for an attribute from a pull down list.

For example, imagine a teacher who is organizing lesson plans in VKB. After performing a search on the topic of "solar system," a set of VKB objects is created along with the metadata available from the source library such as Publisher, Grade Level, Subject, 


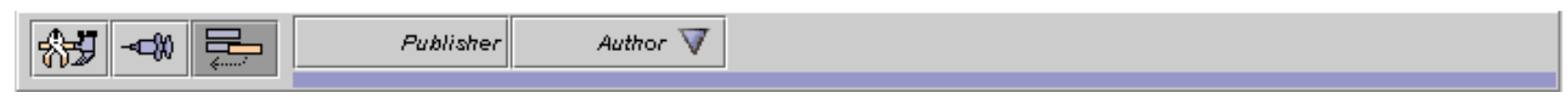

Figure 3: Metadata Applicator prior to extracting attributes

\begin{tabular}{|l||l||l||l||l||}
\hline ofy & Publisher & Author $\nabla$ & Grade_level Resource_t... & collections $\nabla$ \\
\hline
\end{tabular}

Figure 4: Metadata Applicator after extracting attributes

etc. The teacher has also created a group of VKB objects representing lesson plans that she has previously prepared. She wants to attach the same attributes to her lesson plans. So she selects an object returned from her search and chooses to extract attributes. The Metadata Applicator incorporates the attributes from the selected object in the list of available applicator buttons. A single button press causes the Metadata Applicator state to change from that seen in Figure 3 to that of Figure 4. Values imported for the Publisher attribute do not include her name, so she adds herself as a value that can be used for her lesson plans. Now she has a way to quickly add metadata to the set of lesson plans that she "published."

The VKB Suggestion Manager also provides users assistance in applying metadata to information objects. The suggestion system does heuristic evaluation of the workspace as users work. When presenting a suggestion, a small icon moves across the bottom of the workspace. The motion catches users' attention without destroying the context of their work. An attribute suggestion is visible at the bottom of Figure 2. The suggestions remain at the bottom of the screen and eventually fade away if they are not addressed. Users can choose to accept, deny or ignore suggestions. Unlike many suggestion systems, the Suggestion Manager maintains all suggestions in a suggestion history so users can go back and deal with suggestions at their leisure.

Most suggestions can also be implemented automatically by the system. If the suggestion system notices that a set of attributes are being added repeatedly to each information object in a group, the system might offer to do this application for the rest of the objects. Also, it may analyze a group of objects and note that one object does not have an attribute that the rest of the objects in that group have and offer to add that attribute.

\subsection{Embedded History}

The visual languages used to express emergent interpretations or document characteristics evolve over time. Because of this, objects may be given particular visual features early in a task but this expression becomes ambiguous later as the meaning of those visual features change. Since people rarely go back to make the information space completely consistent, this can lead to the inability to interpret or misinterpretations later in the task. Also, when more than one person works on a workspace or when long periods of time pass between uses of a workspace, memory of what a particular visual feature means fades. Consider the case of librarians sharing a table and creating piles of materials for later action. When other librarians see the table, will they be able to figure out the semantics of the piles on the table? Unlike the library table where you cannot go back and see how a particular pile was created, embedded history allows users to go back and see the evolution of the workspace. Additionally, the history events provide an indication of age and frequency of use, much like a book indicating its age and use by its physical condition.

For these reasons, VKB includes an embedded history mechanism similar to Reeves' work on INDY [13] and Hayashi's temporallythreaded workspaces [6]. Users may rewind, replay, or step through the process leading to the current state. The history toolbar is seen below the main visual attributes toolbar in Figure 2. The buttons on the left act like a VCR for playing through the history. The slider in the middle shows where the displayed state is in the event list and allows the user to quickly move to specific states in the history. On the right is the timestamp for the event that resulted in the workspace displayed. Additionally, the user can access a list of the sessions or can ask for particular events on any information object or collection. More information on the VKB history mechanism can be found in [16].

As part of authoring, history events can be grouped and events or groups of events can be meaningfully labeled. As users try to comprehend the information space using history, they can search the history events for events by a particular user or in a particular time period. The search function allows more rapid access into the vast amount of history events that accrue over time.

\section{STUDY}

The goal of VKB is to support the skimming, selection, organization, and interpretation of information from a variety of sources. To understand the impact of VKB on this task, we performed a comparative study of users coping with results from multiple data sources. We chose to compare the work practices and results of using VKB to using the tools commonly used today - the Web browser and an editor or word processor.

When preparing information obtained through on-line searches to share with others, most people will create a text document with the organized information. During this process, they may copy links and text into the document. To analyze this practice we asked subjects to act as a reference librarian, organizing links found through two on-line searches for a teacher that wants to prepare a class lesson on ethnomathematics.

\subsection{Study Design}

16 subjects (13 males and 3 females) were recruited from students and staff at a large university. The subjects ranged in age from 24 to 41. All subjects had more than two years of experience using a computer. The subjects were split into two groups. One group used VKB and a Web browser to complete the task (8 subjects). The control group used a Web browser and an editor of their choosing (Microsoft Word or WordPad) and the folder structure of the Windows operating system (8 subjects). 
All subjects were presented with the same 40 links. Twenty links were the result of a search for ethnomathematics with the National Science Digital Library search engine, and twenty were the results of a search with Google. For VKB subjects, the links were presented in two collections created using the integrated search feature. The control subjects were given the links in two web pages as returned by the Google search and the NSDL search. Static versions of these searches were created to prevent the set of links subjects used from changing during the course of the study.

Subjects were told they should keep relevant links and discard the others. They were told that they could choose to add text, notes, or other information to the documents or to their categories, in order to help the teacher understand their organization. All subjects used Microsoft's Internet Explorer to browse the content of the links.

The VKB subjects had no prior experience using VKB and were all given a brief (10 minute) training session to explain the features of the application. All subjects provided demographic information prior to the task, filled out a questionnaire after the task, and took part in a brief open-ended interview following the questionnaire. During the task we used screen capture software to observe subject work behaviors.

\subsection{Results}

Table 1 presents the results of the answers to the questionnaire. Additionally, we analyzed the screen manipulation playback of each subject's activity and the final products of their organization.

Responses to questionnaire items Q1 and Q2 (see Table 1) were collected using a Likert scale where 1 was strongly disagree, 3 was neutral, and 5 was strongly agree. Items A1 through A12 were the results of analyses of the videos and of subjects' final links organization. Items A9-A12 represent the percentage of subjects performing a certain operation. The first two columns in the table represent the arithmetic mean for subjects in the VKB group and the control group. The third column represents the probability associated with a two-sample unequal variance t-test.
Users in the VKB group were able to better organize the items according to their intention. To the question (see Q1 in Table 1) "I was able to organize everything as I wanted," VKB users replied with an average of 3.63 on a Likert scale, while the average for users in the control group was $2.6(\mathrm{p}=0.064)$.

Similarly users in VKB thought that (Q2) "It will be easy for someone else to understand the way I organized the documents" with an average of 4.13 vs. an average of 3.25 in the control group $(\mathrm{p}=0.132)$.

All users organized the links classifying them in different categories. They chose names for categories such as definition and background, research, books on ethnomathmatics, online resource, etc.

VKB users created collections, often using different colors for the background of the collections, and dragged and dropped reference objects into the collections. They all used the title of the collection to describe the content. Most users (75\%) created two levels of collections and sub collections. One user only created one level of collections and one user three levels. A typical organization with VKB is shown in Figure 2.

A common technique used by all VKB subjects, was to create collections, title them with the name of the documents category, choose a color for them, and drag and drop links into it. Often users organized the documents in two different phases. During the first phase they would create top level categories, they would then heap links inside them. During the second phase they would reorganize each top category, splitting the links into subcategories. Finally, subjects would rearrange links inside each collection to appear more orderly. As an example, Figure 6 illustrates this process for one user.

All users in the control group created a single text file to represent their organization. They would visit the web page, copy and paste the link into the text editor, and occasionally add some lines of text to describe the link. Figure 6 shows a typical result. To structure these results, users created categories using headings and

Table 1: Study Results

\begin{tabular}{|c|c|c|c|}
\hline & VKB & Control & T-TEST (p) \\
\hline Q1: I was able to organize everything as I wanted. & 3.63 & 2.63 & 0.064 \\
\hline Q2: It will be easy for someone else to understand the way I organized the documents. & 4.13 & 3.25 & 0.132 \\
\hline A1: $\quad$ Time spent on the task in minutes & 52.88 & 43.00 & 0.315 \\
\hline A2: Number of links kept & 34.63 & 18.38 & 0.003 \\
\hline A3: Number of links kept from NSDL & 17.13 & 8.13 & 0.002 \\
\hline A4: $\quad$ Number of links kept from Google & 17.50 & 10.25 & 0.015 \\
\hline A5: Number of collections & 9.63 & 5.00 & 0.062 \\
\hline A6: Number of top level collections & 4.75 & 4.00 & 0.506 \\
\hline A7: Number of levels of collections & 2.00 & 1.38 & 0.032 \\
\hline A9: Percentage of subjects in group that added personal comments & 0.00 & 37.50 & 0.080 \\
\hline A10: Percentage of subjects in group that copied and pasted text from web & 12.50 & 50.00 & 0.124 \\
\hline A11: Percentage of subjects in group that processed links in the order presented & 12.50 & 62.50 & 0.043 \\
\hline A12: Percentage of subjects in group that changed links or added ne & 25.00 & 50.00 & 0.335 \\
\hline
\end{tabular}



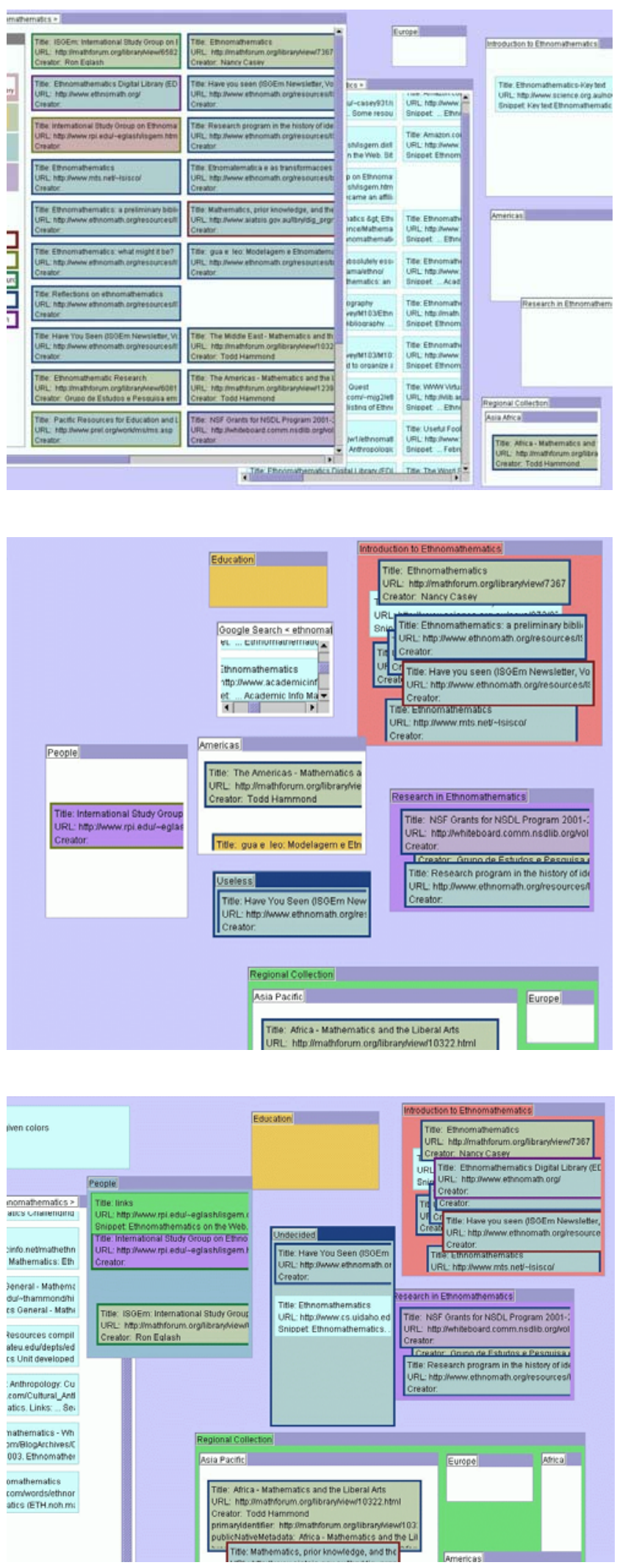

Figure 5: A portion of the workspace for one VKB user early (top), midway through (middle), and finished (bottom)

\author{
What is ethnomathematics? \\ http://www.cs.uidaho.edu/ casev931/seminar/ \\ ethno.html \\ How to pronounce it?

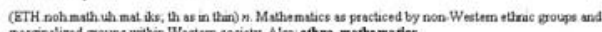

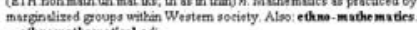 \\ $\rightarrow$ edino mathe matical and \\ http://www.wordspy.com/words/ethnomathe \\ matics.asp \\ Ethnomathematics in Internet \\ http://www.dm.unipi.it/ jama/ethno/ \\ Ethnomathematics Digital Library (EDL) \\ http://www.ethnomath.com/

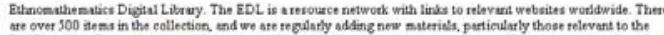

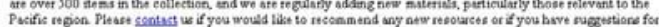 \\ ingroving the broweing or seveching fuactions.
}

\section{Figure 6: Example of organization using a word processor}

subheadings, and grouped links together under those headings. In the control group we use the number of headings and subheadings to count their collections. The total number of collections (two collections one inside the other are counted as two,) is significantly different in the two groups. VKB users used an average of 9.63 collections, while the control group used an average of $5.00 \quad(p=0.062)$. VKB users also used more sub collections: while the number of top collections is similar in both groups, the average number of levels of collections is 2.00 in VKB and 1.38 in the control group (see in Table 1 A5, A6, A7.)

Textual expression was much more common in the control group than those using VKB. None of the users in VKB added personal comments to the links. Only two subjects added a legend with some explanation on how colors were used. Some users in the control group (37.5\%) added personal comments, such as "This site provides resources for teachers and students doing research projects." Users occasionally integrated the link with text copied and pasted from the Web. Only $12.5 \%$ of VKB users did this, while $50.00 \%$ of the control group copied text from the web page. One user copied two pages of text related to a single link.

All subjects were instructed to select the links that they thought were relevant, and to discard the ones that they thought were not useful. While users in VKB kept on average 34.63 links, users in the control group only kept 18.38 of them (A2, $p=0.003$.) A comparison between the number of links kept from NSDL and Google showed little difference. VKB users kept on average 17.13 from NSDL and 17.50 from Google, while subjects in the control group kept 8.13 from NSDL and 10.25 from Google.

Another difference between the VKB users and the control group was the ordering of their activity. The screen captures of the tasks show that $62.5 \%$ of the users in the control group followed the order in which the links were originally presented. Only $12.5 \%$ of the VKB group followed the original order.

Many subjects in the control group (50.00\%) changed the original links or added new links. Sometimes they would follow one of the given links, and browse from there to other web pages that they would include in the document with their organization. Only two subjects in VKB changed any of the original links. In one case a link pointed to the abstract of a document, and in the abstract page there was a link to the actual document. One subject substituted 
the link to the abstract page with the link to the page containing the document itself. Another VKB subject added a couple of links found via browsing.

Subjects were not given a time limit and the resulting difference in the time they took to organize the links is not significant (A1).

The questionnaire also contained freeform questions including: "What kind of information would you want to express that you couldn't express with this approach?," and "What would help you to organize the documents better?". VKB users requested better navigation (3 of the 8), links to part of a document (2), methods to express relationships among objects (1), and the ability to include an object in multiple collections (1). The control group requested more annotation features (3), more flexibility in organizing links (2), a way to rank the links (1), an automated way to format the document (1), a way to keep track of the links already annotated (1), the ability to provide different graphical ways to present results (1), a more efficient way to import information from the web pages into the document (1), and the ability to express relationships (1).

To the question "What was the meaning of the colors in your organization, if any", asked only to the VKB group, five subjects answered that the color indicate different categories. VKB subjects were asked "What did you find the most beneficial feature of VKB" and $75 \%$ of the people answered the "collections". $25 \%$ of the users wrote that they liked the way objects could be moved around.

\subsection{Discussion}

Observing how people cope with documents once they have been retrieved provides insight into both the potential for spatial hypertext to support this activity and issues for the current design of VKB. The basic features of spatial hypertext - the interpretation of documents via direct manipulation and visual and spatial expression - were valued by those using VKB. VKB users created larger and more complex organizations of documents than those in the control group. Issues for VKB include the unwillingness of users to change initial content and visualizations.

\subsubsection{Direct manipulation of documents}

Overall users in the VKB group felt that they were able to better organize the items according to their intention (A1), and they were more confident that their organization would be understood by someone else (A2). In large part, this seems to be due to the ease of manipulating the documents relative to one another. While the control group had to cut and paste text and URLs in order to go about their task, the VKB users could just move the document objects around.

This interpretation is confirmed by several users in the VKB group saying it was nice to be able to move things around at the end of the task. One user said he liked to "be able to put ideas as in post-its, and move them around.” Moving the document objects around allowed users to group documents and visualize their progress in the task.

Collections were particularly valued by VKB users. When asked what the most beneficial feature of VKB was, most users (75\%) said collections. The combination of direct manipulation and collections allowed users to extend on their experience with computer desktops and window systems. Collections were also how the VKB users assigned classification metadata - labeling a collection and placing objects in it meant the items fit into that category. One VKB user asked for the ability to place document objects in multiple collections to indicate multiple categorizations of the documents.

One nice feature of VKB is that the search feature creates a collection of objects, each one representing a link, ready to be manipulated. One user in the control group expressed the need to have a more efficient way to import information from the web pages into the document.

\subsubsection{More complex organization}

Users of VKB were able to deal with more material and used a more structured organization. They kept a much higher number of links (35 vs 20), organized the links in more collections (9.6 vs. 5.0), and used a more complex structure (2 level structure vs. 1.4). There could be several explanations why VKB users were able to deal with more material.

The search feature of VKB creates a collection in which each link reference is embodied into an object. This object can be manipulated directly, dragged and dropped in the space, and in and out of different collections. This makes it easy to reorganize links and to move them from one category to another. Reorganization of links in the control group instead was made by selecting a few lines of text, and copying and pasting them in different position in the text.

Moreover in VKB each object in the original search result is moved to the new location/category, and this helps to keep track of the links already classified. One user in the control group said that he needed "a way to keep track of the links already annotated.” This also implies that while users in VKB were free to process the links in the desired sequence, people using the web search results and the editor had to process the links in the given order to make it easier to keep track of them. In this way each link that is not categorized immediately is more easily left behind. VKB instead affords incremental classifications of links. Some subjects created a temporary collection, with links that they were not sure how to classify (Figure 7). Later, they went back to this collection and created subcollections to classify these remaining miscellaneous links. Users in the control group left these links behind in the web page and never went back to them (they had no way to identify them,) and they end up discarding them.

\subsubsection{Less flexibility in modifying original data}

People in the control group were more likely to add information to their organization and modify the original links. 38\% of subjects in the control group added personal comments, while none did in VKB. They also more easily copied and pasted text from the web. $50 \%$ of people in the control group added or modified links, versus only $25 \%$ in VKB group. This integration of original source materials with annotation and new material was part of what made the model of the library table so appealing. VKB subjects did not use the drag-and-drop or copy-and-create functionality for rapidly getting parts of documents into a workspace. Future work on VKB needs to include a greater emphasis on integrating note-taking and annotation. 


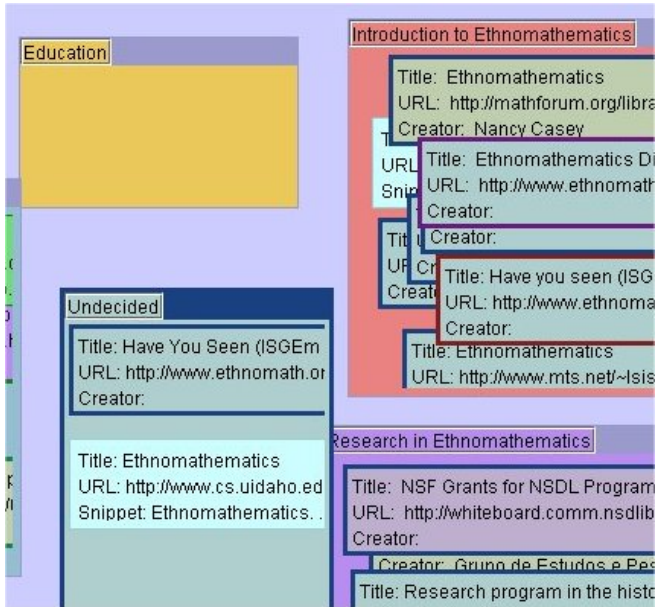

Figure 7: Example of a temporary collection created during the organization task

Not surprisingly, adding or modifying text is easier with a text editor, but at the same time people in VKB showed a bigger reluctance in modifying the original content. In VKB, it was easier to move things around, to organize them, than to modify or add text to them.

The visualization of metadata that was meant to provide added value to the search results from NSDL seems to have created a barrier to users creating their own visual languages using color. Even when users felt there was little value in the visualization, they left the objects as is rather than repurposing color to express additional features of documents. This result is in sharp contrast to our earlier experiences when no initial visualization was provided [10]. In those experiences, color was used as frequently as collections to categorize documents. This poses a design challenge for VKB - how can initial visualizations be presented such that users do not view them as fixed?

\subsubsection{In summary}

The results indicate substantially different work practices between the VKB users and the browser/editor users. VKB provided an easy way to organize references/links to documents. Users found it natural to directly manipulate document objects in space and create categorizations via collections. Document objects created directly from NSDL and Google searches meant less effort for collecting documents but relatively more effort for collecting pieces of documents. Control subjects were more likely to include resources they found by browsing from the search results. These results are likely impacted by the relative inexperience of $\mathrm{VKB}$ users when compared to the control subject's use of familiar tools.

VKB users brought up issues of how their results would fit into their existing work. The reference librarian who took part in the study wanted a way to point to bibliographic entries in EndNote or a similar database in order to fit VKB into her current work practice. Similarly, VKB users asked about the ability to share information during collaborative tasks.

\section{FUTURE WORK}

The results of the study indicate directions for further work as we attempt to build tools to support the interpretive activities of comprehension, annotation, and organization within the information life cycle. Manipulation of the physical information resources includes the piling and arranging of materials on the table and the annotation of those materials with highlighters, marginalia, Post-It notes, etc. More work is necessary on the creation and use of sub-document objects, designs to make ephemeral visualizations, and methods for transitioning visual interpretations into useful metadata.

\subsection{Sub-document objects}

Information objects in spatial hypertext can be pointers to documents available via the Web or file system as well as chunks of text that are copied and pasted into the workspace. Currently, there is no way in VKB to create a reference to part of an external document. While VKB allows for chunks of text and images to be dragged into the workspace, there is no automatically generated reference back to the original source. Such references can be attached to a static piece of a document, as is the case in Microsoft's OneNote product [11] or they can be "live" as in Hunter Gatherer [14] - that is change as the content of the original page changes.

\subsection{Ephemeral visualization}

An unexpected result of the study was the tendency for VKB subjects to avoid changing color and other visual attributes assigned automatically at import based on metadata. Given the high value of color for expressing categorizations in prior studies and experiences with spatial hypertext, there is a clear need to generate visualizations that users are willing to modify or to leave the users with more visual attributes that are not part of the initial visualization. One possibility is to use smaller visual cues attached to document objects. Another option is to include a toggle switch so users can switch between the system-assigned visual attributes and a user-defined freeform visualization.

\subsection{From visual representation to metadata}

The study task was not meant to motivate the addition of formal metadata and so did not evaluate the Metadata Applicator. Further studies will be performed to observe metadata authoring to determine what combination of collections, visual attributes, spatial arrangement, and applicators are preferred. As we understand user preferences, we can improve the metadata suggestions to build on the Suggestion Manager to be more proactive with useful metadata. In the longer-term, we plan to integrate VITE's ability to express formal mappings between visual and metadata representations into VKB [7].

\section{CONCLUSIONS}

Digital libraries exist to support a variety of information tasks. Many of these tasks involve a repeated cycle of location, comprehension, and modification of library materials. We are building tools to support this longer-term activity of digital library patrons using spatial hypertext.

As part of this effort, we have added functionality related to embedded search and metadata application to the Visual Knowledge Builder. The ability to search the NSDL and Google within VKB provides an opportunity to observe the creation of personal and group collections out of public digital libraries. VKB's Metadata Applicator is meant to make authoring metadata less tedious. By automatically extracting metadata attributes and 
values from user-specified exemplars, the Metadata Applicator populates buttons and pull-down menus for the rapid assignment of metadata to other documents.

To understand how the use of spatial hypertext for such tasks changes existing practice, we compared the skimming, culling, and organization of documents in VKB to the common tools of a Web browser and word processor. This study showed that users found the direct manipulation of documents and the ability to create labeled collections to be highly valued. VKB users felt better able to express themselves and more confident that their expressions would be understood by others. On the other hand, VKB users were less willing to select pieces of original documents or provide more substantial annotation of documents. Also, presented with an initial visualization of documents, users were less willing to express their interpretations visually than seen in prior experiences with spatial hypertext.

Our results indicate a value to information workspaces that integrate digital library resources with user interpretation. Future research into the use of spatial hypertext for personal and group digital libraries will need to emphasize the creation of subdocument objects, ephemeral visualizations, and methods for moving from visual to metadata representations. Results from these efforts will better support the work practices currently visible at the library desk or table.

\section{ACKNOWLEDGEMENTS}

This work was supported in part by grant DUE 0226321 from the National Science Foundation. We appreciate the comments of John Leggett and Cathy Marshall on earlier drafts of this paper.

\section{REFERENCES}

[1] Buchanan, G., Blandford, A., Jones, M., and Thimbleby, H. Spatial Hypertext as a Reader Tool in Digital Libraries. In Proceedings of the 2002 Workshop on Visual Interfaces to Digital Libraries. JCDL 2002, 13-24.

[2] Cousins, S. B., Paepcke, A., Winograd, T., Bier, E., and Pier, K. 1997. The digital library integrated task environment (DLITE). In Proceedings of ACM Digital Libraries '97 Conference. ACM Press, New York, NY, 142-151.

[3] diSessa, A., and Abelson, H. (1986), Boxer: a reconstructible computational medium. Communications of the ACM, 29, 9, 859-868.

[4] Fischer, G., Henninger, S., Redmiles, D. Cognitive Tools for Locating and Comprehending Software Objects for Reuse, Thirteenth International Conference on Software Engineering (Austin, TX), IEEE Computer Society Press, ACM, IEEE, Los Alamitos, CA, May 1991, pp. 318-328.

[5] Fischer, G., McCall, R., Ostwald, J., Reeves, B., and Shipman, F., Seeding, Evolutionary Growth, and Reseeding: Supporting the Incremental Development of Design Environments. In Human Factors in Computing Systems: Proceedings of ACM CHI '94, 1994, pp. 292-298.

[6] Hayashi, K., Nomura, T., Hazama, T., Takeoka, M., Hashimoto, S., and Gudmundson, S. Temporally-threaded Workspace: A Model for Providing Activity-based
Perspectives on Document Spaces. Proceedings of ACM Hypertext ' 98 Conference, 1998, pp. 87-96.

[7] Hsieh H., and Shipman, F. Manipulating Structured Information in a Visual Workspace. In Proceedings of ACM Conference on User Interface Software and Technology 2002, 2002, pp. 217-226.

[8] Lagoze, C., Arms, W., Gan, S., Hillmann, D., Ingram, C., Krafft, D., Marisa, R., Phipps, J., Saylor, J., Terrizzi, C., Hoehn, W., Millman, D., Allan, J., Guzman-Lara, S. and Kalt, T., Core services in the architecture of the national science digital library (NSDL), Proceedings of the second ACM/IEEE-CS joint conference on Digital libraries, July 1418, 2002, Portland, Oregon, USA

[9] Marshall C.C., and Shipman, F.M. Spatial Hypertext: Designing for Change. Communications of the ACM, 38, 8 (August 1995), 88-97.

[10] Marshall C.C., and Shipman, F.M. Effects of Hypertext Technology on the Practice of Information Triage. Proceedings of ACM Hypertext '97 Conference, 1997, pp. 167-176.

[11] Microsoft. Introducing Microsoft Office OneNote 2003. http://office.microsoft.com/assistance/preview.aspx?AssetID =HA010731591033\&CTT=98, visited on Jan. 15, 2004.

[12] Rao R., Pedersen. J.O., Hearst, M.A., Mackinlay, J.D., Card, S.K., Masinter, L., Halvorsen, P. and Robertson, G.C., Rich interaction in the digital library, Communications of the ACM, v.38 n.4, p.29-39, April 1995

[13] Reeves, B. Supporting Collaborative Design by Embedded Communication and History in Design Artifacts. Ph.D. Dissertation, Department of Computer Science, University of Colorado, 1993.

[14] Schraefel, M.C., Modjeska, D., Wigdor, D., Zhu, Y. Hunter Gatherer: Interaction Support for Within-Web-page Collection Making. In Proceedings of the WWW2002 Conference.

[15] Shipman, F., Furuta, R., and Marshall, C. "Generating WebBased Presentations in Spatial Hypertext", Proceedings of the ACM Intelligent User Interfaces Conference, Orlando, Florida, (Jan. 6-9, 1997), pp. 71-78.

[16] Shipman, F. and Hsieh, H. Navigable History: A Reader's View of Writer's Time. New Review of Hypermedia and Multimedia, 6 (2000).

[17] Shipman, F., and McCall, R. 1999. Supporting incremental formalization in the Hyper-Object Substrate. ACM Transactions on Information Systems, 17, 2 (April 1999), 199-227.

[18] Shipman, F., Moore, J.M., Maloor, P., Hsieh, H., and Akkapeddi, R. Semantics Happen: Knowledge Building in Spatial Hypertext. In Proceedings of the 2002 Conference on Hypertext. ACM Press, New York, NY, 2002, 25-34.

[19] Sumner, T. and Dawe, M. Looking at Digital Library Usability from a Reuse Perspective. In Proceedings of 2001 Joint Conference on Digital Libraries (JCDL 2001). ACM Press, New York, NY, 2001, 416-425. 